\title{
The Long-Term Outcomes after Radical Prostatectomy of Patients with Pathologic Gleason 8-10 Disease
}

\author{
Dan Lewinshtein, Brandon Teng, Ashley Valencia, \\ Robert Gibbons, and Christopher R. Porter
}

\author{
Section of Urology and Renal Transplantation, Virginia Mason Medical Center, 1100 Ninth Avenue, C7-URO, Seattle, \\ WA 98101, USA \\ Correspondence should be addressed to Dan Lewinshtein, danlewinshtein@gmail.com
}

Received 7 April 2011; Revised 20 June 2011; Accepted 21 July 2011

Academic Editor: Steven Joniau

Copyright ( $\odot 2012$ Dan Lewinshtein et al. This is an open access article distributed under the Creative Commons Attribution License, which permits unrestricted use, distribution, and reproduction in any medium, provided the original work is properly cited.

\begin{abstract}
Background. We explored the long-term clinical outcomes including metastases-free survival and prostate cancer-specific survival (PCSS) in patients with pathologic Gleason 8-10 disease after radical prostatectomy (RP). Methods. We report on 91 patients with PCSS data with a median followup of 8.2 years after RP performed between 1988 and 1997. Cox regression and Kaplan-Meier analysis were used to evaluate year of surgery, pathologic stage, and surgical margin status as predictors of PCSM. Results. Median age was 65 years (IQR: 61-9), and median PSA was $9.7 \mathrm{ng} / \mathrm{ml}$ (IQR: 6.1-13.4). Of all patients, 62 (68.9\%) had stage T3 disease or higher, and $48(52.7 \%)$ had a positive surgical margin. On multivariate analysis, none of the predictors were statistically significant. Of all patients, the predicted 10-year BCR-free survival, mets-free survival, and PCSS were 59\% (CI: 53\%-65\%), 88\% (CI: 84\%92\%), and 94\% (CI: 91\%-97\%), respectively. Conclusions. We have demonstrated that cancer control is durable even 10 years after RP in those with pathologic Gleason 8-10 disease. Although $40 \%$ will succumb to BCR, only 6\% of patients died of their disease. These results support the use of RP for patients with high-risk localized prostate cancer.
\end{abstract}

\section{Introduction}

Since the advent of widespread prostate-specific antigen (PSA) testing in the late 1980s and early 1990s, PC detection has increased with a concomitant downward-shift in stage $[1,2]$. In addition to PSA, the introduction of the anatomic radical retropubic prostatectomy (RP) [3, 4], breakthroughs in radiotherapy delivery [5], and systemic chemotherapeutic agents [6] have resulted in a 30\% mortality reduction [7]. Despite the stage shift and treatment improvements, $15 \%$ of contemporary patients will present with highrisk localized prostate cancer [8]. Contemporary high-risk disease is generally defined by high Gleason score rather than elevated PSA or advance stage due PSA testing and digital rectal examination $[8,9]$. Unfortunately, $50 \%$ of patients with high-risk disease will succumb to biochemical failure within 10 years $[10,11]$.
Although there are no randomized trials that support the use of RP for patients with high-risk disease, recent retrospective studies lend credence to the use of RP as an effective therapy for this group of patients $[12,13]$. The renewed interest is based on multiple benefits of surgery. First, it provides excellent local control. Second, it better defines the extent of disease than biopsy alone [14]. Third, with prostate removal, PSA failures can be more easily detected. Fourth, radiation can be given in the adjuvant or salvage setting whereas surgery after radiation is associated with high complication rates [15].

In that context, we hypothesized that patients with pathologic Gleason 8-10 disease may have better long-term clinical outcomes after RP than previously thought. Thus we explored the long-term clinical outcomes including metastases-free survival and prostate cancer-specific survival (PCSS) in these high-risk patients after RP. 


\section{Materials and Methods}

We retrospectively analyzed charts of patients who underwent radical prostatectomy (RP) between 1988 and 1997 at Virginia Mason Medical Center (VMMC). No patient received neoadjuvant therapy. One of the authors (R. Gibbons) logged clinical and pathological data into a prospective database from 1988 to 1999. After 1999, the records were maintained electronically with institutional review board (IRB) approval. PSA testing began in 1988 at VMMC. All patients were operated between 1988 and 1997. We subselected for a cohort of 91 patients that had pathologic Gleason 8 disease or higher and had postoperative PSA data available. Of all patients, $66(72.5 \%)$ underwent radical retropubic prostatectomy (RRP) and $25(27.5 \%)$ underwent radical perineal prostatectomy (RPP). All specimens were evaluated by VMMC pathologists. Specimens were processed as half mount specimens and serially sectioned at $5 \mathrm{~mm}$ intervals. Alternate $5 \mathrm{~mm}$ sections were fixed in formalin and were paraffin embedded. From 1988 to 1992 pathological tumor stage was recorded according to the Whitmore-Jewett classification and later converted to the 1992 AJCC staging guidelines [16, 17] From 1992 to 1997 the 1992 AJCC staging system was used for clinical and pathological staging.

From 1992 to 1997 tumors were routinely classified according to the Gleason grading system [18]. Before 1992 tumor grade was recorded as well differentiated (I), moderately differentiated (II), and poorly differentiated (III). To recode these data we followed the paradigm outlined by Roehl et al., in which well-differentiated tumors are classified as Gleason sum 3, moderately differentiated tumors are assigned Gleason sum 6, and poorly differentiated tumors are assigned Gleason sum 9 [19]. Positive surgical margins were recorded as presence of cancer cells against the inked resection margin.

Serum PSA testing was initiated at VMMC in 1988. Since that time patients were followed at least quarterly for 2 years, then at least biannually for 2 years, and then at least annually. Biochemical recurrence was defined as PSA greater than $0.1 \mathrm{ng} / \mathrm{mL}$. Metastases were diagnosed based on technetium-99m-based bone scintigraphic studies, and computed tomography cross-sectional imaging was used in equivocal cases. Adjuvant or salvage hormonal and/or radiotherapy were delivered according to individual surgeon preference. Adjuvant therapy was defined as adjunctive radiotherapy in the absence of PSA recurrence (PSA < $0.1 \mathrm{ng} / \mathrm{mL}$ ) given within 6 months of surgery. Neoadjuvant hormonal therapy was defined as hormone delivery prior to surgery. Cause of death was ascertained according to detailed chart review or was obtained from the VMMC cancer registry. The cancer registry uses links with the Washington State Death Certificate Office. PC must be the first listed cause of death on the certificate for a patient to be classified as having died of PC.

Prostate cancer-specific mortality was analyzed with univariate and multivariate Cox regression models based on preoperative and operative factors. Predictors included year of surgery, 1992 AJCC pathological stage, and surgical margin status.
TABLE 1: Demographics and pathologic and clinical outcomes.

\begin{tabular}{lc}
\hline Characteristic & No. (IQR) \\
\hline Median followup in years & $8.2(4.5-12.5)$ \\
Median age in years & $65(61-69)$ \\
Median pre-op PSA & $9.7(6.1-13.4)$ \\
Pathologic tumor volume & $5.3(3.0-12.0)$ \\
Pathologic Gleason sum & No. $(\%)$ \\
Gleason 8 & $68(74.7)$ \\
Gleason 9 & $22(24.2)$ \\
Gleason 10 & $1(1.1)$ \\
Pathologic stage & \\
$\quad$ pT2 & $28(31.1)$ \\
pT3/4 & $62(68.9)$ \\
Positive margins & $48(52.7)$ \\
Lymph node dissection and node status & \\
$\quad$ Nx & $34(37.4)$ \\
$\quad$ N0 & $49(53.8)$ \\
N1 & $6(6.6)$ \\
N2 & $2(2.2)$ \\
Adjuvant radiation & $10(11.0)$ \\
Salvage radiation & $10(11.0)$ \\
Neoadjuvant ADT & $9(9.9)$ \\
Balvage ADT & $19(20.9)$ \\
Metastatic disease & $33(36.3)$ \\
PCSM & $8(8.8)$ \\
\hline ADtal no. patients & $9(9.9)$ \\
Parcal recurrence & $91(100)$ \\
\hline
\end{tabular}

ADT: androgen deprivation therapy.

Actuarial analyses addressed the outcomes of PSA recurrence, distant recurrence, PCSS, and overall survival. In analyses of PCSS, patients without evidence of progression were censored at the time of last followup. A biochemical recurrence event for the purposes of Kaplan-Meier analysis was defined as a PSA of $0.1 \mathrm{ng} / \mathrm{mL}$ or the delivery of radiotherapy or hormonal therapy after later than 6 months after surgery. Kaplan-All statistical tests and figures were performed with S-PSS (2009) and statistical significance was set at 0.05 .

Patients who died of other causes were censored at time of death. PSA recurrence-free data were censored if radiotherapy and/or hormonal therapy were delivered before PSA recurrence.

\section{Results}

Clinical and pathologic characteristics are shown in Table 1. The median followup was 8.2 years (interquartile range [IQR]: $4.5-12.5$ years). The median age of the 91 person cohort was 65 years (IQR: 61-69 years) and the median PSA was $9.7 \mathrm{ng} / \mathrm{mL}$ (IQR: 6.1-13.4). At time of pathological analysis after RP, $62(68.9 \%)$ had stage T3 disease or higher, $23(25.3 \%)$ had pathologic Gleason 9 or higher, and 48 $(52.7 \%)$ had a positive surgical margin. 
TABLE 2: Binary logistic regression for prostate cancer-specific mortality.

\begin{tabular}{|c|c|c|c|c|}
\hline Characteristics & Univariate hazard ratio $(95 \% \mathrm{CI})$ & $(P$ value $)$ & Multivariate hazard ratio (95\% CI) & $(P$ value $)$ \\
\hline Pre-op PSA & $1.038(0.970-1.112)$ & 0.278 & $1.042(0.953-1.139)$ & 0.366 \\
\hline Gleason ( 8 versus $9-10)$ & $0.830(0.160-4.312)$ & 0.825 & $1.087(0.106-11.146)$ & 0.944 \\
\hline Stage (pT3/4 versus pT2) & $4.000(0.476-33.645)$ & 0.202 & $0.627(0.032-12.429)$ & 0.759 \\
\hline Margin status & $1.905(0.446-8.136)$ & 0.384 & $4.943(0.342-71.440)$ & 0.241 \\
\hline XRT received & $1.912(0.433-8.442)$ & 0.392 & $3.529(0.356-34.987)$ & 0.281 \\
\hline ADT received & $3.207(0.790-13.007)$ & 0.103 & $0.703(0.050-9.948)$ & 0.795 \\
\hline Node status & $1.257(0.127-12.419)$ & 0.845 & $2.127(0.059-77.249)$ & 0.680 \\
\hline
\end{tabular}

$\mathrm{XRT}=$ adjuvant or salvage radiotherapy; ADT: hormone use for relapse.

TABle 3: Kaplan-Meier actuarial estimates of BCR-free survival, metastases-free survival, overall survival, and prostate cancer specific survival (PCSS).

\begin{tabular}{lcccc}
\hline Cohort & Overall cohort & pT2/margin - & pT3 or margin + & pT3 \& margin + \\
\hline No at risk/5-year BCR-free survival (CI) & $90 / 0.69(0.064-0.74)$ & $22 / 0.84(0.76-0.90)$ & $25 / 0.65(0.55-0.75)$ & $42 / 0.64(0.56-0.68)$ \\
No at risk/5-year mets-free survival (CI) & $90 / 0.94(0.91-0.97)$ & $22 / 1.00(1.00-1.00)$ & $25 / 0.96(0.92-1.00)$ & $42 / 0.89(0.84-0.94)$ \\
No at risk/5-year overall survival (CI) & $90 / 0.96(0.94-0.98)$ & $22 / 1.00(1.00-1.00)$ & $25 / 1.00(1.00-1.00)$ & $42 / 0.92(0.88-0.96)$ \\
No at risk/5-year PCSS (CI) & $90 / 0.97(0.95-0.99)$ & $22 / 1.00(1.00-1.00)$ & $25 / 1.00(1.00-1.00)$ & $42 / 0.95(0.91-0.99)$ \\
No at risk/10-year BCR-free survival (CI) & $47 / 0.59(0.53-0.65)$ & $13 / 0.77(0.66-0.88)$ & $13 / 0.65(0.55-0.75)$ & $21 / 0.47(0.38-0.56)$ \\
No at risk/10-year mets-free survival (CI) & $61 / 0.88(0.84-0.92)$ & $16 / 1.00(1.00-1.00)$ & $19 / 0.96(0.92-1.00)$ & $26 / 0.77(0.69-0.85)$ \\
No at risk/10-year overall survival (CI) & $65 / 0.84(0.79-0.89)$ & $16 / 0.85(0.75-0.95)$ & $20 / 0.83(0.74-0.92)$ & $29 / 0.85(0.79-0.91)$ \\
No at risk/10-year PCSS (CI) & $65 / 0.94(0.91-0.97)$ & $16 / 1.00(1.00-1.00)$ & $20 / 0.88(0.80-0.96)$ & $29 / 0.95(0.91-0.99)$ \\
No at risk/15-year BCR-free survival (CI) & $29 / 0.52(0.45-0.59)$ & $8 / 0.77(0.66-0.88)$ & $9 / 0.45(0.31-0.59)$ & $12 / 0.47(0.38-0.56)$ \\
No at risk/15-year mets-free survival (CI) & $37 / 0.88(0.84-0.92)$ & $9 / 1.00(1.00-1.00)$ & $12 / 0.96(0.92-1.00)$ & $16 / 0.77(0.69-0.85)$ \\
No at risk/15-year overall survival (CI) & $40 / 0.69(0.62-0.76)$ & $9 / 0.85(0.75-0.95)$ & $12 / 0.74(0.62-0.86)$ & $19 / 0.59(0.48-0.70)$ \\
No at risk/15-year PCSS (CI) & $40 / 0.80(0.73-0.87)$ & $9 / 1.00(1.00-1.00)$ & $12 / 0.78(0.66-0.90)$ & $19 / 0.73(0.62-0.84)$ \\
\hline
\end{tabular}

Table 2 shows the univariate and multivariate Cox regression model predicting prostate cancer-specific mortality. On both univariate and multivariate analysis, none of predictors remained statistically significant $(P>0.05)$.

Figure 1 graphically displays Kaplan-Meier estimates of BCR (a), metastases (b), overall survival (c), and PCSS (d) stratified by pathologic stage and surgical margin status. There was a trend for mean times to BCR $(P=0.081)$, and metastatic disease $(P=0.060)$, to be different between pT2/margin negative patients and pT3/margin positive patients.

Table 3 provides Kaplan-Meier actuarial estimates for time to BCR, metastases, overall survival and PCSS. Of all patients, the predicted 10-year BCR-free survival, mets-free survival, and PCSS were 59\% (CI: 53\%-65\%), 88\% (CI: 84\%-92\%), and 94\% (CI: 91\%-97\%), respectively. Specifically, the predicted 10 -year BCR-free rate was significantly better in those with organ-confined margin negative disease (pT2) than in those with locally advanced (pT3) margin positive disease (77\% (CI: $66 \%-88 \%$ ) versus $47 \%$ (CI: $38 \%-$ $56 \%)$ ). The predicted 15-year PCSS was significantly better in those with organ-confined margin negative disease (pT2) than in those with locally advanced (pT3) margin positive disease (100\% (CI: 100\%-100\%) versus 73\%(CI: 62\%-84\%)).

\section{Discussion}

We have demonstrated in a cohort with a median followup time of 8.2 years that cancer control is durable even 10 years after RP in those with pathologic high-grade disease. Although, $41 \%$ of patients developed BCR by 10 years, only $12 \%$ of patients in this extremely high-risk group progressed to distant metastases, and just $6 \%$ of patients actually died of their disease (Table 3).

When put into context, 59\% of all patients with pathologic Gleason 8 disease or higher and $47 \%$ of patients with pathologic Gleason 8 disease or higher, pT3 stage and margin positive disease were cured of their disease (no BCR within $10 \mathrm{yr}$ ) with primary RP (Table 3 ). However, cure was not achieved solely with surgery. Of all patients, 11 received solely postoperative radiotherapy, 10 received long-term hormonal therapy, and 9 received both postoperative radiotherapy and long-term hormonal therapy. Clearly, this is a group of patients that will require multimodal therapy to achieve robust durable outcomes.

Our 10-year actuarial disease-specific mortality estimate (6\%) was similar to other long-term RP series, including the UCLA group (8\%) [20] and Hull et al. (2.4\%) [21], and compares favorably with reports that have examined locally advanced disease specifically $[12,22]$.

It is intriguing that the classical predictors of outcome such as pathological stage and surgical margin status did not reach statistical significance in our multivariate analysis. However, the multivariate effect of these variables on PCSS was not assessed in most other long-term outcome series except that of Stephenson et al. [23]. However, stage was a significant predictor on multivariate analyses of BCR after 


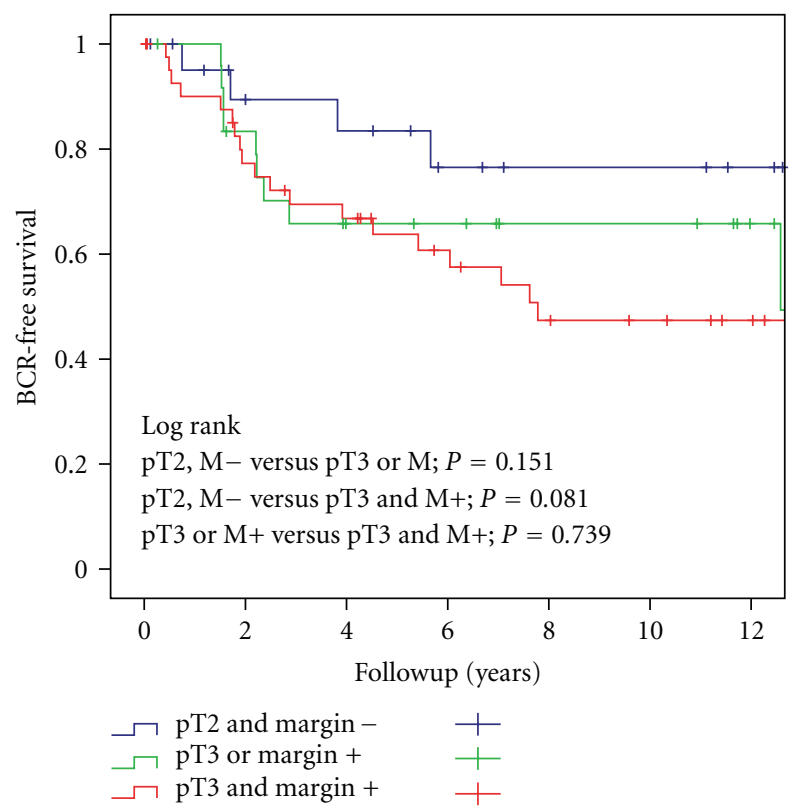

(a)

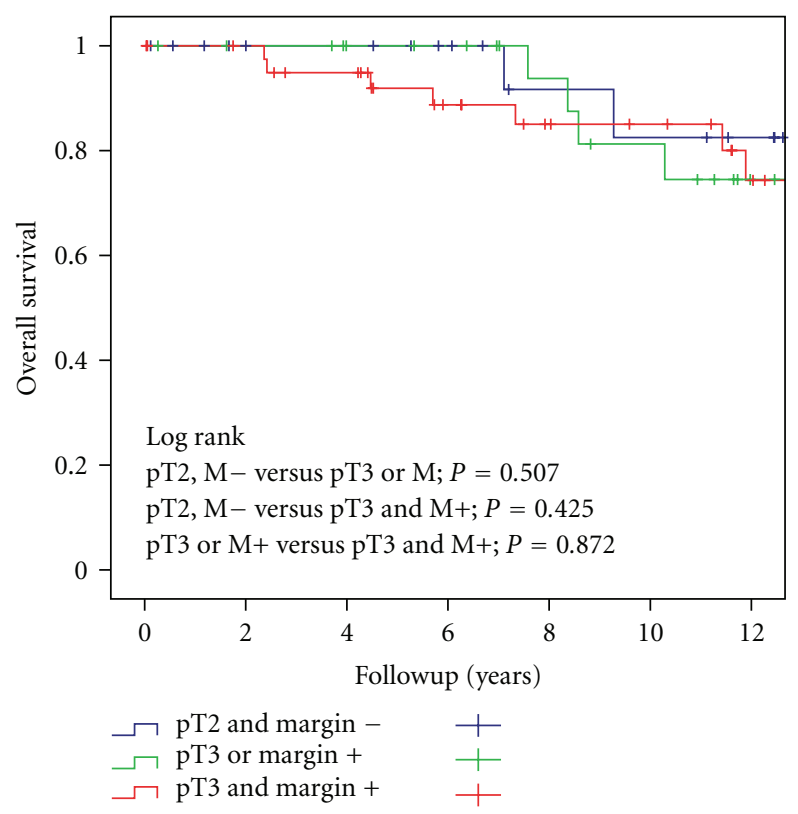

(c)

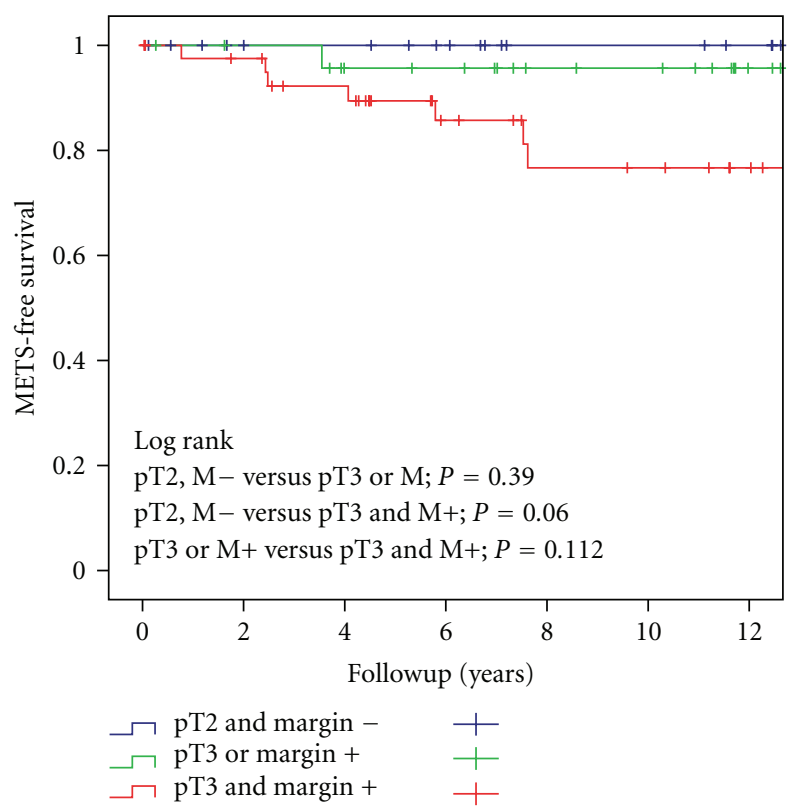

(b)

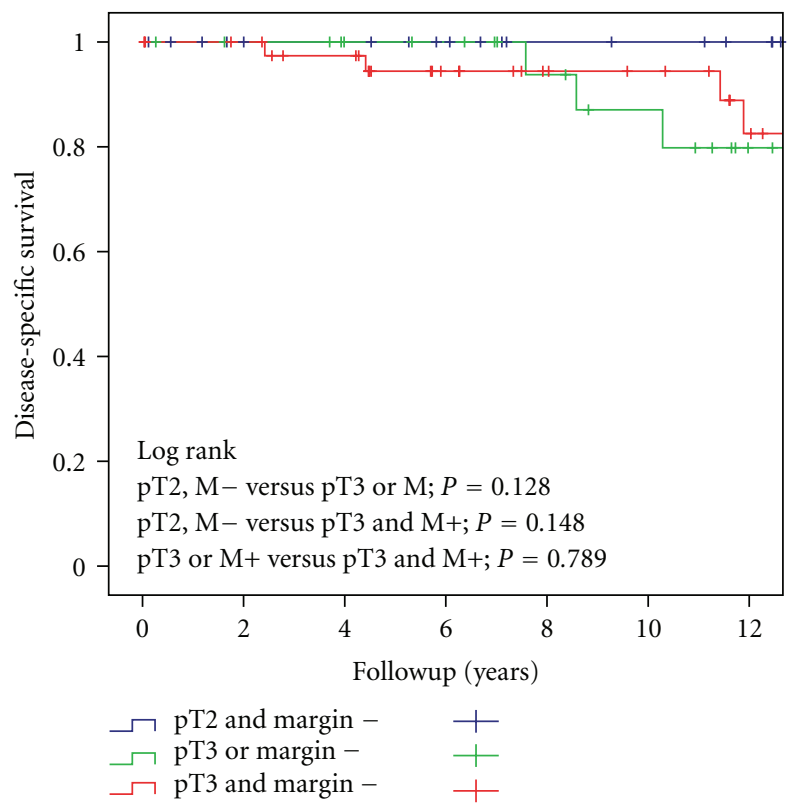

(d)

Figure 1: Kaplan-Meier estimates of BCR (a), distant recurrence (b), overall survival (c), and disease-specific survival (d) according to stage and surgical margin status.

$\mathrm{RP}$ in other series with long-term followup $[19,21]$ as was surgical margin status [21]. Thus, the positive predictor status of these classic variables with regard to BCR, but their inability to predict long-term PCSM suggest that they may not be important with regard to long-term oncologic control. This assumption will require further study in other longterm followup RP series.

The results of our study must be interpreted within the strengths and limitations of our study. First, our data derive from a single center over 20 years, and involve multiple surgeons. Thus, patient selection and surgical technique certainly differed and possibly may have introduced variation in outcome [24]. Moreover, patients underwent two surgical techniques, namely, RPP and RRP, which may have affected outcome. However, the literature suggests that there is no difference between RPP and RRP oncologic outcomes [25]. Second, we recognize that there is no centralized pathologic review and that therefore contemporary Gleason scores may well be ascribed a higher value [14]. However, all pathology was read at a single tertiary referral center 
with a high level of GU pathology expertise. Moreover, we relied upon the original pathology report from VMMC to establish the histological differentiation. Gleason scores were not routinely recorded before 1992, and therefore, we relied upon the paradigm used by Roehl to assign Gleason scores to patients undergoing surgery before 1992 [19]. Finally, postoperative use of radiation and androgen deprivation therapy were given at the discretion of the treating physician and may have introduced substantial bias into the interpretation of the results.

\section{Conclusions}

In summary, we have demonstrated in a cohort with a median followup time of 8.2 years that cancer control is durable even 10 years after RP in those with pathologic Gleason 810 disease. Although, $41 \%$ of patients developed BCR by 10 years, only $12 \%$ of patients in this high-risk group progressed to metastases, and just $10 \%$ of patients died of their disease. Taken together, these long-term oncologic results support the use of RP for patients with high-risk localized prostate cancer.

\section{References}

[1] W. J. Catalona, J. P. Richie, F. R. Ahmann et al., "Comparison of digital rectal examination and serum prostate specific antigen in the early detection of prostate cancer: results of a multicenter clinical trial of 6,630 men," Journal of Urology, vol. 151, no. 5, pp. 1283-1290, 1994.

[2] S. Soh, M. W. Kattan, S. Berkman, T. M. Wheeler, and P. T. Scardino, "Has there been a recent shift in the pathological features and prognosis of patients treated with radical prostatectomy?" Journal of Urology, vol. 157, no. 6, pp. 2212-2218, 1997.

[3] P. C. Walsh and H. Lepor, "The role of radical prostatectomy in the management of prostatic cancer," Cancer, vol. 60, no. 3, pp. 526-537, 1987.

[4] A. Bill-Axelson, L. Holmberg, F. Filén et al., "Radical prostatectomy versus watchful waiting in localized prostate cancer: the Scandinavian prostate cancer group-4 randomized trial," Journal of the National Cancer Institute, vol. 100, no. 16, pp. 1144-1154, 2008.

[5] A. Widmark, O. Klepp, A. Solberg et al., "Endocrine treatment, with or without radiotherapy, in locally advanced prostate cancer (SPCG-7/SFUO-3): an open randomised phase III trial," The Lancet, vol. 373, no. 9660, pp. 301-308, 2009.

[6] I. F. Tannock, R. de Wit, W. R. Berry et al., "Docetaxel plus prednisone or mitoxantrone plus prednisone for advanced prostate cancer," The New England Journal of Medicine, vol. 351, no. 15, pp. 1502-1512, 2004.

[7] A. Jemal, R. Siegel, E. Ward, Y. Hao, J. Xu, and M. J. Thun, "Cancer statistics, 2009," CA Cancer Journal for Clinicians, vol. 59, no. 4, pp. 225-249, 2009.

[8] M. R. Cooperberg, D. P. Lubeck, S. S. Mehta, and P. R. Carroll, "Time trends in clinical risk stratification for prostate cancer: implications for outcomes (data from CaPSURE)," Journal of Urology, vol. 170, no. 6, pp. S21-S25, 2003.

[9] C. J. Kane, J. C. Presti Jr., C. L. Amling, W. J. Aronson, M. K. Terris, and S. J. Freedland, "Changing nature of high risk patients undergoing radical prostatectomy," Journal of Urology, vol. 177, no. 1, pp. 113-117, 2007.

[10] A. W. Partin, J. Yoo, H. B. Carter et al., "The use of prostate specific antigen, clinical stage and Gleason score to predict pathological stage in men with localized prostate cancer," Journal of Urology, vol. 150, no. 1, pp. 110-114, 1993.

[11] W. J. Catalona and D. S. Smith, "Cancer recurrence and survival rates after anatomic radical retropubic prostatectomy for prostate cancer: intermediate-term results," Journal of Urology, vol. 160, no. 6, pp. 2428-2434, 1998.

[12] S. Loeb, E. M. Schaeffer, B. J. Trock, J. I. Epstein, E. B. Humphreys, and P. C. Walsh, "What are the outcomes of radical prostatectomy for high-risk prostate cancer?" Urology, vol. 76, no. 3, pp. 710-714, 2010.

[13] S. A. Boorjian, R. J. Karnes, L. J. Rangel, E. J. Bergstralh, and M. L. Blute, "Mayo clinic validation of the D'amico risk group classification for predicting survival following radical prostatectomy," Journal of Urology, vol. 179, no. 4, pp. 13541360, 2008.

[14] T. Fukagai, T. Namiki, H. Namiki, R. G. Carlile, M. Shimada, and H. Yoshida, "Discrepancies between Gleason scores of needle biopsy and radical prostatectomy specimens," Pathology International, vol. 51, no. 5, pp. 364-370, 2001.

[15] G. T. Gotto, L. H. Yunis, K. Vora, J. A. Eastham, P. T. Scardino, and F. Rabbani, "Impact of prior prostate radiation on complications after radical prostatectomy," Journal of Urology, vol. 184, no. 1, pp. 136-142, 2010.

[16] B. Carter and A. Partin, "Prostate cancer staging systems," in Campbell's Urology, P. C. Walsh, A. B. Retik, E. D. Vaughan Jr., and A. J. Wein, Eds., p. 2526, W. B. Saunders, Philadelphia, Pa, USA, 7th edition, 1998.

[17] O. H. Beahrs, D. E. Henson, R. V. P. Hutter, and B. J. Kennedy, American Joint Committee on Cancer: Manual for Staging of Cancer, J. B. Lippincott, Philadelphia, Pa, USA, 4th edition, 1992.

[18] D. F. Gleason and G. T. Mellinger, "Prediction of prognosis for prostatic adenocarcinoma by combined histological grading and clinical staging," Journal of Urology, vol. 111, no. 1, pp. 58-64, 1974.

[19] K. A. Roehl, M. Han, C. G. Ramos, J. A. V. Antenor, and W. J. Catalona, "Cancer progression and survival rates following anatomical radical retropubic prostatectomy in 3,478 consecutive patients: long-term results," Journal of Urology, vol. 172, no. 3, pp. 910-914, 2004.

[20] H. Zinoke, J. E. Oesterling, M. L. Blute, E. J. Bergstralh, R. P. Myers, and D. M. Barrett, "Long-term (15 years) results after radical prostatectomy for clinically localized (stage T2c or lower) prostate cancer," Journal of Urology, vol. 152, no. 5, pp. 1850-1857, 1994.

[21] G. W. Hull, F. Rabbani, F. Abbas, T. M. Wheeler, M. W. Kattan, and P. T. Scardino, "Cancer control with radical prostatectomy alone in 1,000 consecutive patients," Journal of Urology, vol. 167, no. 2, pp. 528-534, 2002.

[22] S. Loeb, N. D. Smith, K. A. Roehl, and W. J. Catalona, "Intermediate-term potency, continence, and survival outcomes of radical prostatectomy for clinically high-risk or locally advanced prostate cancer," Urology, vol. 69, no. 6, pp. 1170-1175, 2007.

[23] A. J. Stephenson, M. W. Kattan, J. A. Eastham et al., "Prostate cancer-specific mortality after radical prostatectomy for patients treated in the prostate-specific antigen era," Journal of Clinical Oncology, vol. 27, no. 26, pp. 4300-4305, 2009. 
[24] J. A. Eastham, M. W. Kattan, E. Riedel et al., "Variations among individual surgeons in the rate of positive surgical margins in radical prostatectomy specimens," Journal of Urology, vol. 170, no. 6, pp. 2292-2295, 2003.

[25] L. Salomon, O. Levrel, A. de la Taille et al., "Radical prostatectomy by the retropubic, perineal and laparoscopic approach: 12 years of experience in one center," European Urology, vol. 42, no. 2, pp. 104-110, 2002. 


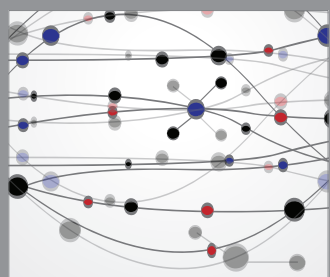

The Scientific World Journal
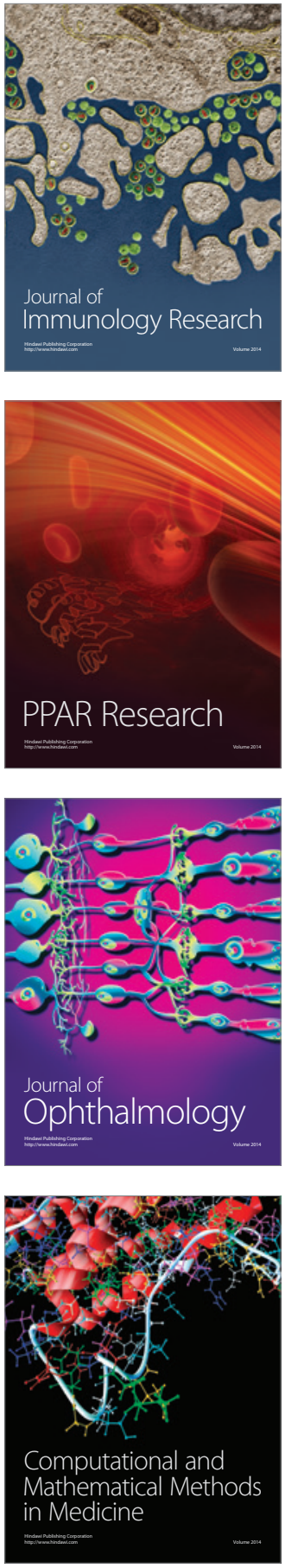

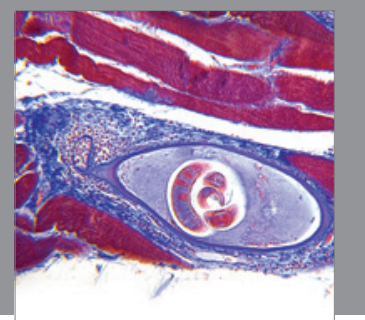

Gastroenterology

Research and Practice
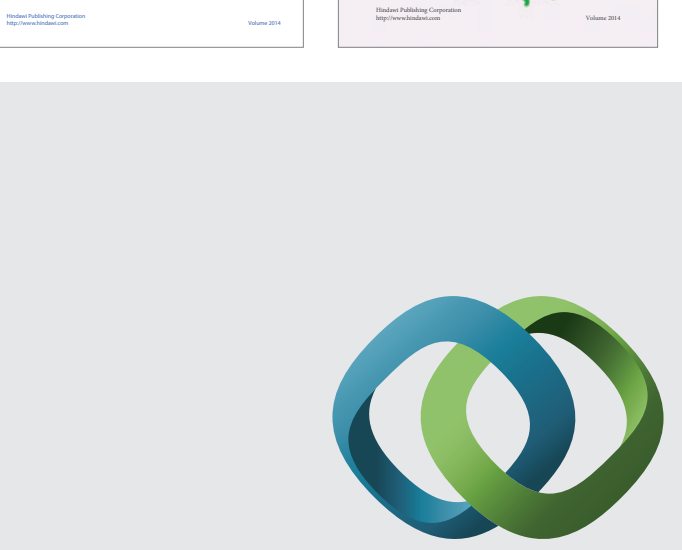

\section{Hindawi}

Submit your manuscripts at

http://www.hindawi.com
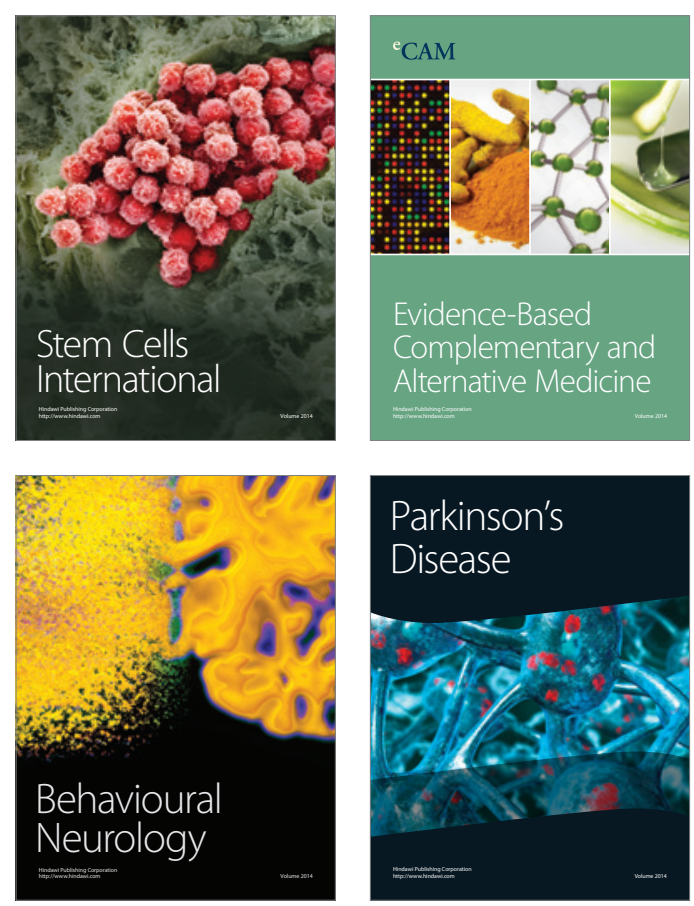

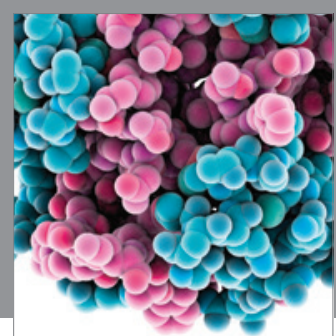

Journal of
Diabetes Research

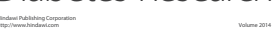

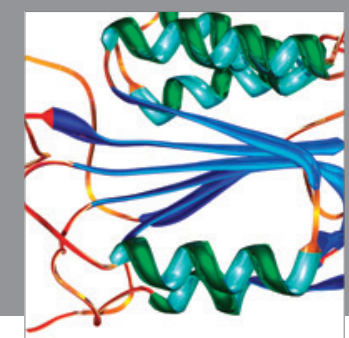

Disease Markers
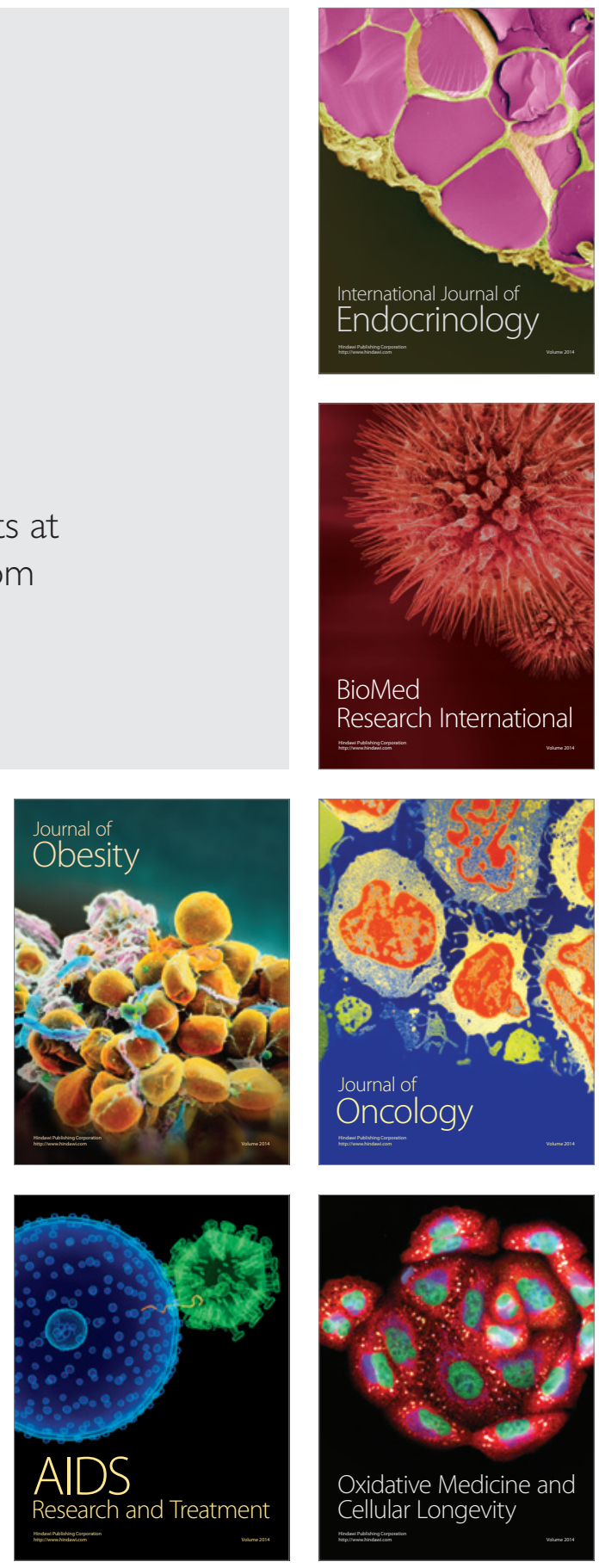